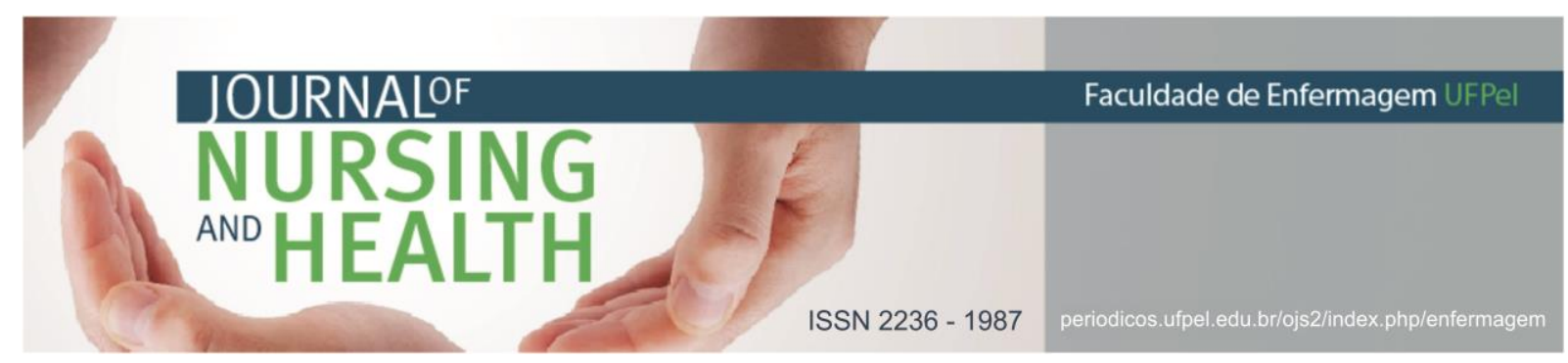

ARTIGO ORIGINAL

\title{
Nascer em Porto Alegre: a realidade obstétrica na capital gaúcha
}

\author{
Being born in Porto Alegre: obstetrical reality in the capital of Rio Grande do Sul
}

Nacer en Porto Alegre: la realidad obstétrica en la capital de Rio Grande do Sul

Aline Alves VELEDA ${ }^{1}$, Marcia Cordeiro da CUNHA ${ }^{2}$, Adriana Finger VITÓRIA ${ }^{3}$, Bruna Vieira OLIVEIRA ${ }^{4}$

\section{RESUMO}

Objetivo: descrever a realidade obstétrica no município de Porto Alegre. Método: pesquisa descritiva exploratória realizada no percurso de uma tese de doutorado. Foram utilizados dados a partir do banco do DATASUS, da Agência Nacional de Saúde Suplementar e do Observatório da cidade de Porto Alegre. Foi realizada uma análise descritiva sobre a realidade obstétrica. Resultados: Observou-se uma cobertura adequada de pré-natal. Identificou-se um percentual de cesarianas nas instituições privadas de até $86 \%$. Ressalta-se que onde as condições de vida são as melhores os percentuais de cesariana variaram de $71 \%$ (Centro) até $56 \%$ (Cristal), identificando o componente socioeconômico determinante. Conclusões: na realidade da capital gaúcha dependendo de onde a mulher mora podese consideravelmente cogitar o tipo de parto que ela terá. Quanto melhor a qualidade de vida e dependendo da região de moradia ela possui maiores chances de ter seu filho por via cirúrgica.

Descritores: saúde suplementar; obstetrícia; políticas públicas.

\begin{abstract}
Objective: this work was aimed to describe the obstetrical care reality in Porto Alegre. Methods: this is a descriptive and exploratory research. Birth information data was retrieved from DATASUS database, property from the National Agency of Additional Health Care, and from Porto Alegre's Observatory. A descriptive analysis was performed on the obstetric reality. Results: Adequate antenatal coverage was observed. A rate of up to $86 \%$ of caesarean section on private hospitals was identified. Rates varied from $71 \%$ (Downtown) to $56 \%$ (Cristal district), where quality of life is better, featuring the socioeconomic component as determinant. Conclusions: in Porto Alegre, it may be possible to predict women's delivery methods according to where they live. Women are more likely to go through C-section when they have better quality of life and depending on which neighborhood they live.

Descriptors: Supplemental health; Obstetrics; Public policies.
\end{abstract}

\section{RESUMEN}

Objetivo: este trabajo tiene como objetivo describir la realidad obstétrica de Porto Alegre. Metodología: posee como metodología la pesquisa descriptiva exploratoria. Fueron utilizados datos referentes a los nacimientos desde el banco de datos DATASUS, de la Agencia Nacional de Salud

\footnotetext{
1 Enfermeira. Doutora em Enfermagem. Professora Adjunta na Universidade Federal de Ciências da Saúde de Porto Alegre, Porto Alegre, Rio Grande do Sul, Brasil. Email: alineav@ufcspa.edu.br

2 Acadêmica de Enfermagem na Universidade Federal de Ciências da Saúde de Porto Alegre, Porto Alegre, Rio Grande do Sul, Brasil. Email: jf-marcia@hotmail.com

${ }^{3}$ Acadêmica de Enfermagem na Universidade Federal de Ciências da Saúde de Porto Alegre, Porto Alegre, Rio Grande do Sul, Brasil. Email: adri.finger@hotmail.com

${ }^{4}$ Enfermeira. Residente na Residência Integrada em Saúde do Grupo Hospitalar Conceição ênfase Atenção Materno Infantil e Obstetrícia, Grupo Hospitalar Conceição, Porto Alegre, Rio Grande do Sul, Brasil. Email: bru.olivi@gmail.com
} 


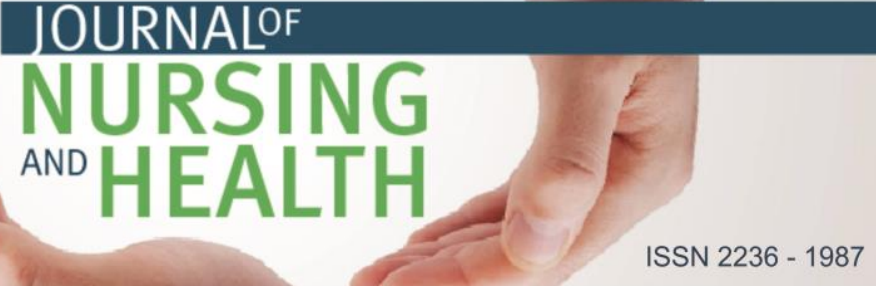

Suplementar y del Observatorio de Porto Alegre. Un análisis descriptivo se realizó. Resultados: Hubo una cobertura adecuada de la atención prenatal. Se identificó un índice del $86 \%$ de cesáreas en las instituciones privadas. Se constató que donde las condiciones de vida son mejores, los porcentuales de cesáreas varían del 71\% (Centro) al 56\% (Cristal), identificando el componente socioeconómico de forma determinante. Conclusiones: en realidad, en la capital gaucha podemos cogitar el tipo de parto que la mujer tendrá, de acuerdo con el lugar donde ella vive. Cuanto mejor la cualidad de vida, y dependiendo de la región donde se encuentra, más chances de tener su hijo por vía quirúrgica. Descriptores: Salud complementaria; Obstetricia; Políticas públicas.

\section{INTRODUÇÃO}

O panorama atual brasileiro sobre as práticas de parto e nascimento sofreu diversas mudanças desde meados do século passado, demonstrando crescente hospitalização, medicalização e mercantilização, e tornando a cirurgia cesariana um modo de dar à luz condizente com a modernidade e a praticidade. ${ }^{1}$ Nascer no Brasil não é nada natural, tanto no setor público, quanto nos estabelecimentos privados, evidenciando uma assistência marcada ora pelo excesso de intervenções desnecessárias, ora pelo uso indiscriminado da tecnologia "préagendada". ${ }^{2}$

Apesar desta realidade estar presente em quaisquer serviços obstétricos do sistema de saúde é no subsetor suplementar/privado que os índices de nascimentos por via cirúrgica, sem recomendações clínicas evidentes, extrapolam as indicações da Organização Mundial de Saúde, exigindo continuamente estudos que possam caracterizar esta situação e indicar formas de mudança possíveis de serem implementadas. Afinal, é evidente que não se pode associar os índices elevados de cirurgia cesariana encontradas na realidade obstétrica atual a uma solicitação ou desejo das mulheres, visto que comprovadamente elas costumam referir o parto normal como a sua primeira opção. ${ }^{3}$
Assim, o que percebe-se é que o direito à informação e a autonomia das mulheres não está sendo respeitado, demonstrando a necessidade da implantação de novas ações estratégicas que envolvam características socioculturais, demográficas e obstétricas, bem como mudanças na formação profissional e na estrutura dos serviços de saúde. ${ }^{4}$ No entanto, para colocar estas mudanças em prática, primeiramente, é preciso conhecer a realidade obstétrica da saúde suplementar setorialmente, identificando as relações existentes entre lugares de vida e lugares de cuidado e delimitando quais ações são mais necessárias a partir das particularidades regionais.

Neste sentido, esse estudo propõe-se a descrever a realidade obstétrica de Porto Alegre, Rio Grande do Sul, a partir de dados apresentados nos anos de 2010 a 2014, com o intuito de contribuir significativamente com a programação em saúde e, principalmente, com a adequação das políticas públicas de atenção maternoinfantil. Acredita-se que, a partir de uma descrição e conhecimento desta realidade pode-se auxiliar em um processo de mudança no modelo de cuidado materno e neonatal garantindo a humanização, o uso de evidências científicas e a redução das cesarianas desnecessárias. 


\section{NURSING \\ AND \\ HEALTH}

ISSN 2236 - 1987

\section{MATERIAIS E MÉTODOS}

Trata-se de uma pesquisa descritiva e exploratória realizada no percurso de uma tese de doutorado desenvolvida na Universidade Federal do Rio Grande do Sul cuja metodologia utilizada foi a etnografia. Neste artigo destaca-se a primeira parte desta pesquisa, a de caracterização, descrição e familiarização com o campo de estudo: a realidade obstétrica de Porto Alegre, Rio Grande do Sul, utilizando-se dados tanto do setor público quando da atenção suplementar à saúde.

Foram utilizados dados referentes aos nascimentos em Porto Alegre a partir do banco de dados do DATASUS, da Agência Nacional de Saúde Suplementar (ANS) e o Observatório da cidade de Porto Alegre (ObservaPOA) ${ }^{5}$. No DATASUS pesquisou-se dados referentes a nascidos vivos, tipo de nascimentos, locais de nascimento e financiamento dos partos e cesarianas. No banco de dados da ANS foram pesquisados operadoras de saúde com registro ativo, taxa de cobertura, número de beneficiários, sexo e idade dos beneficiários e instituições de saúde cadastradas.

No ObservaPOA ${ }^{5}$ foram pesquisados tipo de parto por financiamento, mortalidade infantil, mortalidade materna, pré-natal adequado e regiões do Orçamento Participativo (OP). As regiões do orçamento participativo dividem 0 município de Porto Alegre em 17 áreas, as quais são utilizadas para a definição coletiva de investimentos e serviços específicos necessários para a comunidade. Análises comparativas de saúde são realizadas entre as regiões possibilitando a participação popular nas decisões sobre as políticas públicas específicas para sua região.

Posteriormente foi realizada uma análise descritiva sobre a realidade obstétrica a partir dos achados nos bancos de dado mencionados. Os dados são apresentados em figuras (gráficos e mapas), ambos também disponíveis no ObservaPOA ${ }^{5}$. Essa ferramenta de dados da Prefeitura de Porto Alegre possibilita o cruzamento de algumas informações de saúde, permitindo a análise de informações de forma conjunta.

O período utilizado para coleta dos dados foram os últimos anos disponíveis destes no momento da análise, sendo de 2012 a 2014. Já a coleta de dados foi realizada no ano de 2014 durante a realização de uma tese de doutorado.

Cabe salientar que se trata de uma análise de dados epidemiológicos descritiva e não inclui em sua metodologia o contato direto com sujeitos, utilizando apenas dados secundários. Dessa maneira não houve necessidade de submissão do mesmo em um comité de ética em pesquisa.

\section{RESULTADOS}

A capital possui sete hospitais que oferecem assistência em obstetrícia pelo subsetor suplementar, totalizando 155 leitos não públicos neste setor. Proporcionalmente os nascimentos pelo setor público continuam sendo a maioria e apenas 30\% ocorrem nos hospitais privados. Em 2013, último dado disponível nacionalmente, Porto Alegre contava com 51,60\% dos nascimentos ocorrendo por via cirúrgica. 


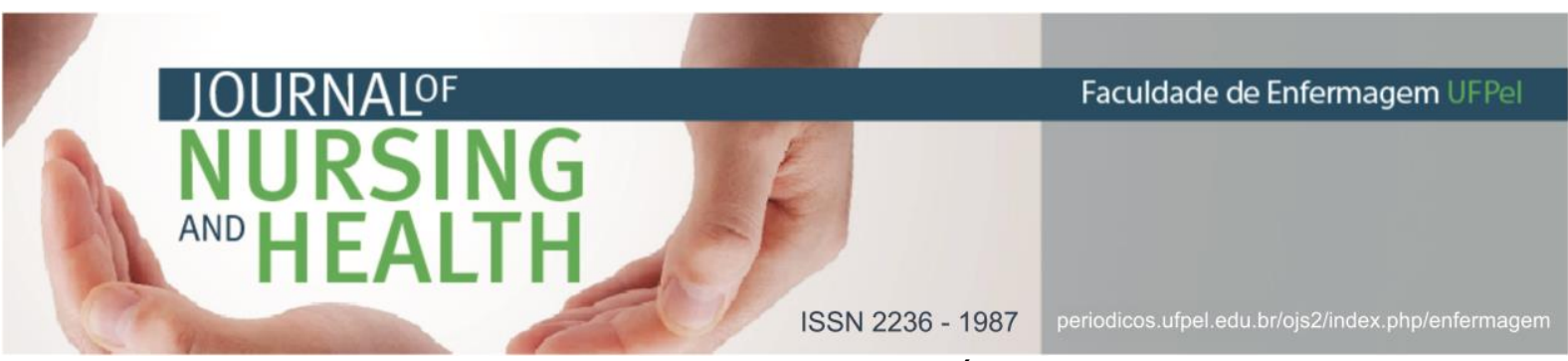

Ao analisar este dado, separando por hospitais públicos e privados, identificou-se um índice discrepante de cesarianas nas instituições privadas, podendo chegar a $86 \%$ do total de nascimentos. Esta realidade nos fez refletir sobre a mortalidade materna e neonatal do município, as quais podem estar sendo influenciadas pelos percentuais de nascimento por cesariana encontrados, visto ser este procedimento indicado em situações de risco materno e neonatal.

Em 2014 identificou-se quatro casos de morte materna em Porto Alegre, sendo que destes três óbitos foram durante a gravidez, parto e aborto. O coeficiente de mortalidade neonatal precoce é de 5,35 e o de mortalidade neonatal tardia de 1,42. De acordo com dados do ObservaPOA ${ }^{5}$, em 2014, 9,38\% das crianças nascidas vivas tinham menos de $2,5 \mathrm{~kg}$, sendo o maior percentual na Região Sul do

município, reconhecidamente a região que possui um dos melhores indicadores em população economicamente ativa e responsáveis com renda maior que dez salários mínimos.

Cabe ressaltar, em relação ao alto índice de cesarianas no município, algumas relações podem ser estabelecidas. Dentre elas destacamos que, apesar deste índice aumentar consideravelmente nos últimos anos, as mortalidades maternas e infantis não sofreram alterações significativas. A mortalidade infantil desde 2009 mantem-se estável apesar do crescimento deste procedimento, já a mortalidade materna apresentou picos em 2012 e uma queda até 2014. Estes dados podem ser observados nas figuras 1 e 2, gerado a partir de um cruzamento possível de ser realizado por meio do ObservaPOA ${ }^{5}$, conforme já mencionado na metodologia:

\section{Porto Alegre}

\section{Gráfico comparativo da evolução percentual dos indicadores selecionados}

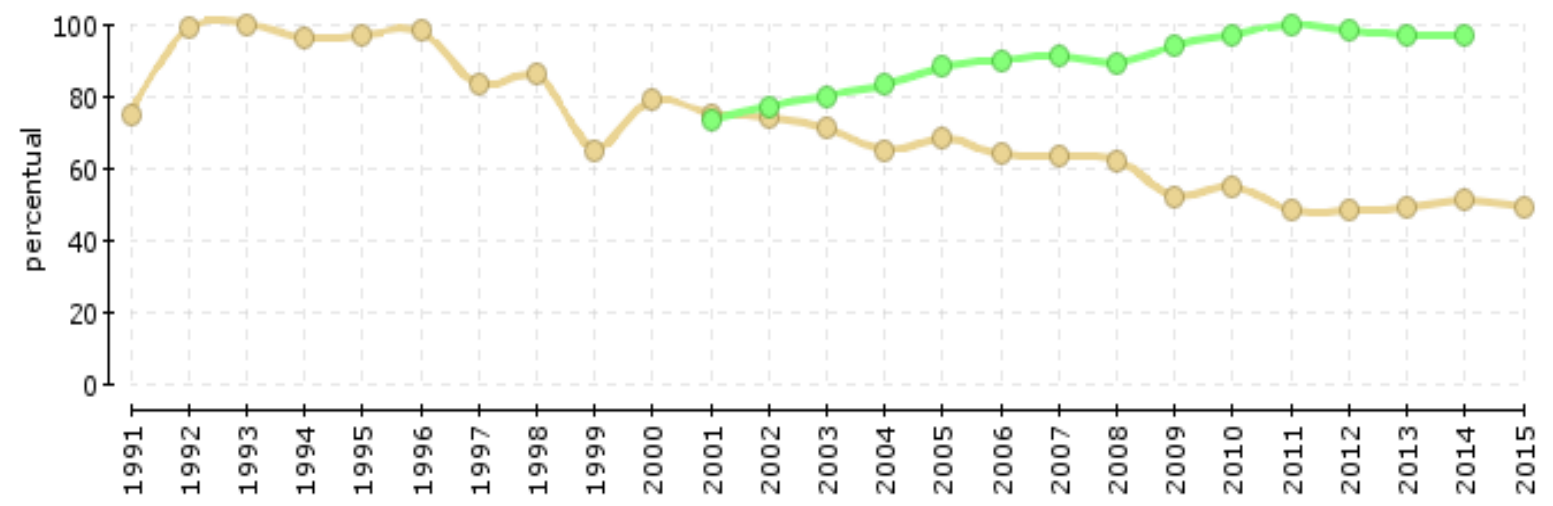

Os valores máximos de cada indicador são representados por $100 \%$.

- Mortalidade infantil

- Parto œsáreo

Figura 1 - Mortalidade infantil e cesarianas em Porto Alegre: série histórica de 1991 até 2012. Fonte: Porto Alegre em Análise, ObservaPOA, $2015^{5}$. 


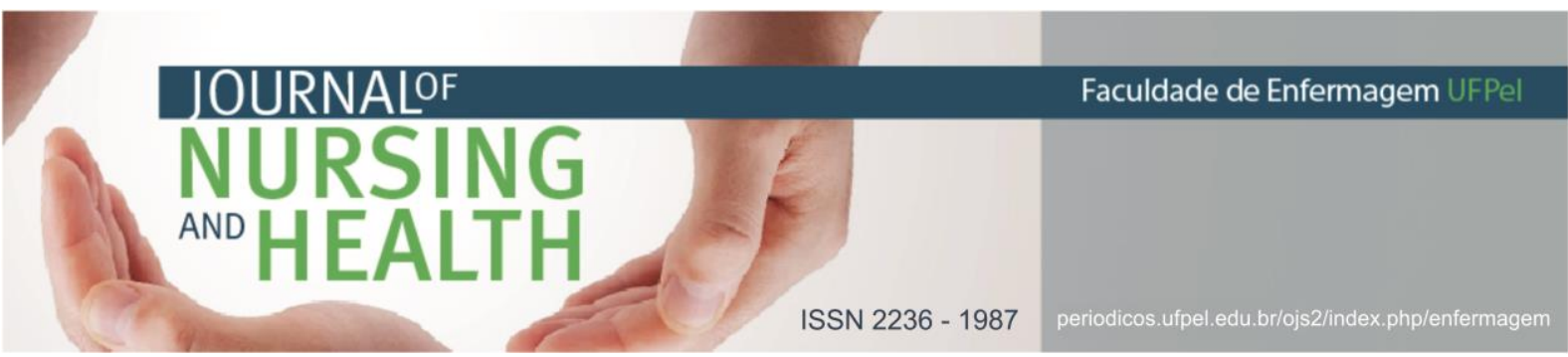

Porto Alegre

Gráfico comparativo da evolução percentual dos indicadores selecionados

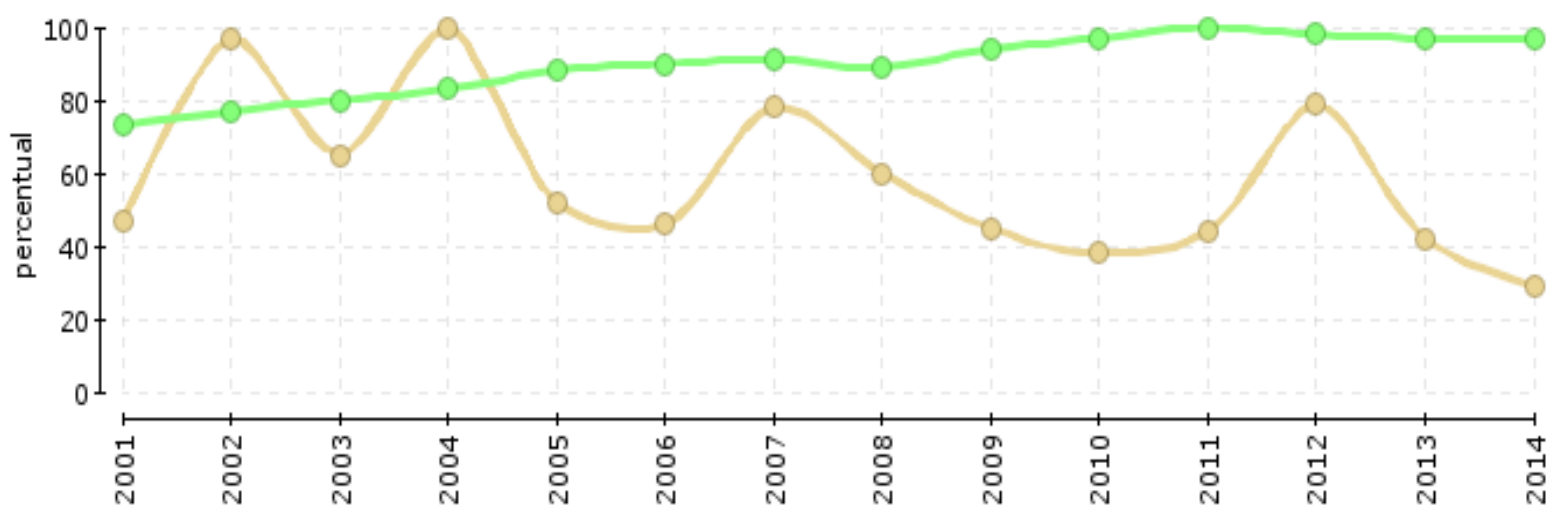

Os valores máximos de cada indicador são representados por $100 \%$.

Mortalidade matema

- Parto œsáreo

Figura 2 - Mortalidade materna e cesarianas em Porto Alegre: série histórica de 1991 até 2012. Fonte: Porto Alegre em Análise, ObservaPOA, $2015^{5}$.

Ainda de acordo com o ObservaPOA 5 , observou-se, de forma geral, percentuais adequados de cobertura pré-natal que Porto Alegre no ano de 2013 , sendo que $73,33 \%$ das mulheres-gestantes realizam sete ou mais consultas durante este período, fator reconhecidamente protetivo para a saúde da gestante e do feto. Não foram encontrados dados estratificados na população atendida exclusivamente pelos serviços suplementares de saúde.

Desde 1989, a administração de Porto Alegre funciona por meio do Orçamento Participativo (OP), um processo dinâmico de participação popular na gestão urbana que vem servindo como modelo para diversos outros municípios em nível mundial. A gestão pelo OP, em busca de maior aproximação com as necessidades locais, implantou uma divisão do município em 17 regiões, facilitando a realização de análises mais próximas das realidades e comparações entre as diversas regiões.

Em relação ao Índice de Desenvolvimento Humano Municipal, as regiões com melhores resultados foram a Centro, Noroeste, Sul e Cristal. Estas mesmas regiões ainda contam com os melhores índices de escolaridade e rendimento médio entre os responsáveis pelo domicílio, maior número de população economicamente ativa e com renda superior a dez salários mínimos. Estes dados referem-se à 2010, último ano analisado no ObservaPOA ${ }^{5}$, mas precisam com urgência serem revistos já que muitas condições municipais modificaram desde esta data.

Considerando-se que nestes locais estão algumas regiões de melhor qualidade de vida e maiores oportunidades no município, resolveuse buscar informações sobre pré-natal, mortalidade infantil e tipo de parto 


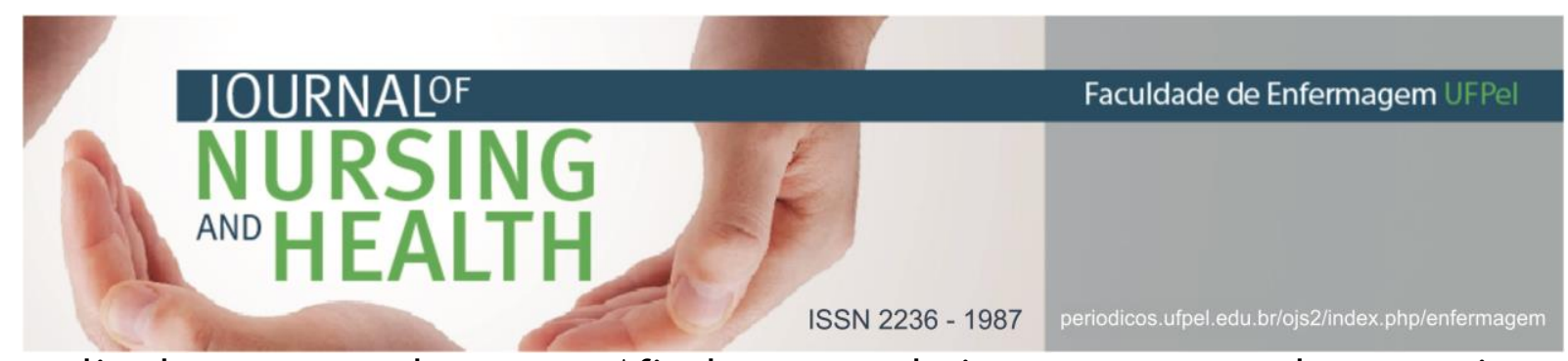

realizado nestes lugares. Afinal, parece claro que, sendo aí a qualidade de vida melhor, poder-se-ia encontrar menores percentuais de gestações de risco e consequentemente menores percentuais de cesarianas.

No entanto, nestas regiões, onde as condições de vida são as melhores na capital, os percentuais de nascimentos ocorridos por cesariana variaram de $71 \%$ no Centro até $56 \%$ na região Cristal. Enquanto que nas regiões com piores rendimentos médios por domicílio, condições de infraestrutura inadequadas e índices de desenvolvimento humano abaixo do esperado, onde possivelmente poderiam ser encontrados os maiores riscos obstétricos, os percentuais de cesariana não chegam a $40 \%$.

Além disso, esta disparidade também aparece quando consideramos o total de pré-natal adequado, onde as regiões mais desenvolvidas possuem melhores percentuais de cobertura pré-natal, chegando a $84 \%$ na região Centro, e as regiões mais pobres apresentam um índice que pode chegar a 59\% na Restinga. Cabe salientar que consideramos aqui como pré-natal adequado o mínimo de seis consultas, iniciando o acompanhamento até o quarto mês de gestação. Isto pode ser observado nas figuras 3 a 5 .

Porto Alegre

Gráfico comparativo da evolução perœentual dos indicadores selecionados

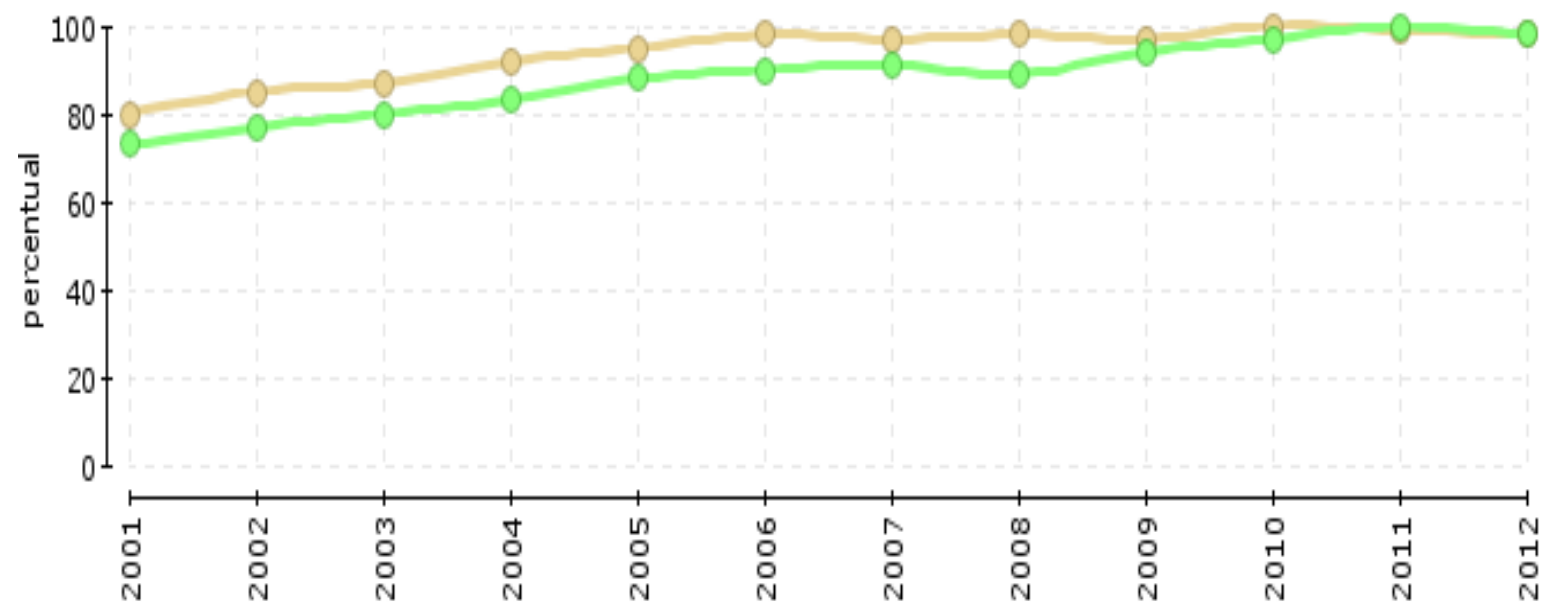

Os valores máximos de cada indicador são representados por $100 \%$.

\section{Pré-natal adequado - Parto cesáreo}

Figura 3- Pré-natal adequado e cesarianas em Porto Alegre: série histórica de 2001 a 2012. Fonte: Porto Alegre em Análise, ObservaPOA - 20155. 


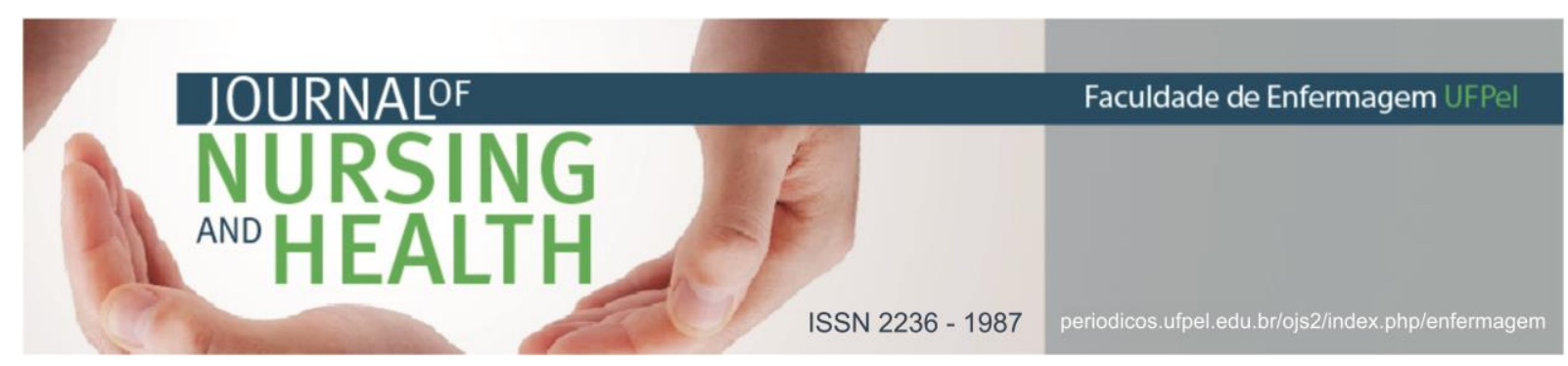

\section{Ano 2012 - Regiões de Porto Alegre}

\section{Fator de Desigualdade}

Entre as Regiões, a proporção entre o melhor e o pior valor, em 2012, foi de 1,43 vezes.

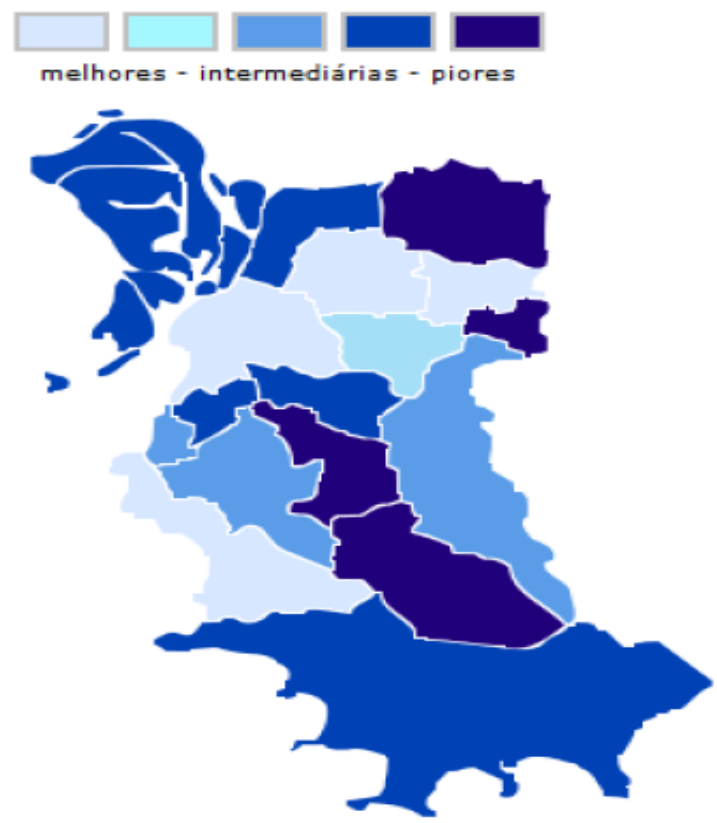

\begin{tabular}{|lrr|}
\hline \multicolumn{1}{|c|}{ Região } & Indicador & V.Absoluto \\
\hline Porto Alegre & 70,41 & 13.699 \\
Centro & 84,24 & 2.186 \\
Noroeste & 84,11 & 1.122 \\
Eixo Baltazar & 75,86 & 968 \\
Sul & 75,79 & 839 \\
Leste & 71,36 & 1.176 \\
Cristal & 69,84 & 264 \\
Lomba do Pinheiro & 69,15 & 769 \\
Centro Sul & 68,27 & 977 \\
Extremo Sul & 67,40 & 339 \\
Partenon & 67,08 & 1.145 \\
Cruzeiro & 66,36 & 651 \\
Humaitá / Navegantes & 66,04 & 453 \\
Ilhas & 64,12 & 84 \\
Norte & 62,26 & 894 \\
Glória & 60,11 & 437 \\
Nordeste & 60,07 & 331 \\
Restinga & 59,00 & 531 \\
\hline
\end{tabular}

Figura 4 - Pré-natal em Porto Alegre: Regiões do Orçamento Participativo.

Fonte: Porto Alegre em Análise, ObservaPOA - $2015^{5}$.

\section{Ano 2012 - Regiões de Porto Alegre}

\section{Fator de Desigualdade}

Entre as Regiões, a proporção entre o melhor e o pior valor, em 2012, foi de 2,12 vezes.

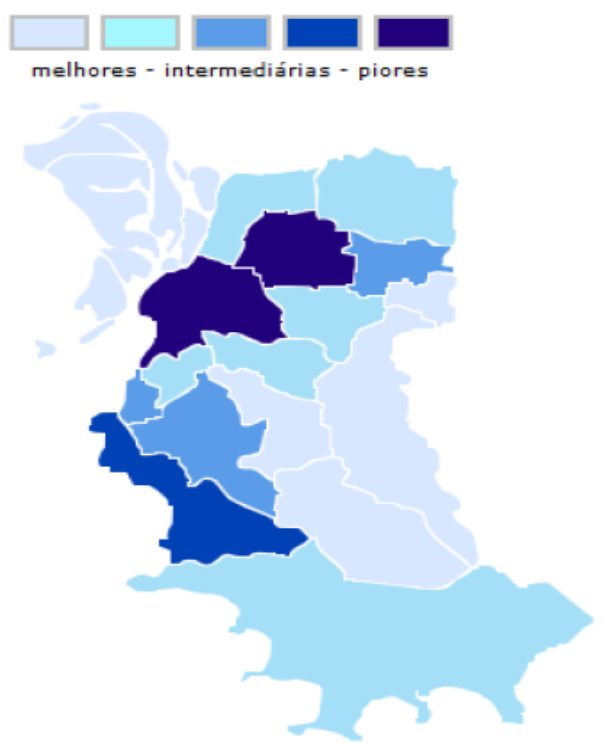

\begin{tabular}{|c|c|c|c|}
\hline \multicolumn{2}{|c|}{ Região } & Indicador & V.Absoluto \\
\hline \multicolumn{2}{|c|}{ Porto Alegre } & 52,23 & 10.161 \\
\hline & Ilhas & 33,59 & 44 \\
\hline$\square$ & Lomba do Pinheiro & 38,76 & 431 \\
\hline 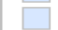 & Nordeste & 39,20 & 216 \\
\hline L & Restinga & 40,56 & 365 \\
\hline 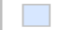 & Glória & 44,43 & 323 \\
\hline L & Partenon & 45,17 & 771 \\
\hline 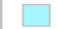 & Leste & 46,18 & 761 \\
\hline 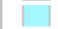 & Cruzeiro & 46,18 & 453 \\
\hline 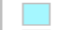 & Extremo Sul & 46,32 & 233 \\
\hline 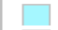 & Norte & 46,38 & 666 \\
\hline 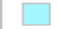 & Humaitá / Navegantes & 50,15 & 344 \\
\hline E & Centro Sul & 52,90 & 757 \\
\hline E & Cristal & 56,88 & 215 \\
\hline & Eixo Baltazar & 58,15 & 742 \\
\hline & Sul & 61,97 & 686 \\
\hline E & Noroeste & 70,61 & 942 \\
\hline & Centro & 71,21 & 1.848 \\
\hline
\end{tabular}

Parâmetro de referência:

Taxas de cesárea maiores que $10 \%$ não estão

associadas com redução de mortalidade materna e neonatal.

Figura 5: Nascimentos por cesariana nas Regiões do Orçamento Participativo.

Fonte: Porto Alegre em Análise, ObservaPOA - 20155. 


\section{DISCUSSÃO}

Partindo-se dos dados apresentados sobre a realidade obstétrica da capital gaúcha, torna-se necessária uma discussão e reflexão sobre como estão acontecendo os nascimentos e os determinantes que possam estar influenciando principalmente nos índices de cesarianas encontrados.

Cesarianas sem indicação clínica constituem um problema de saúde pública não só em Porto Alegre, mas nacionalmente, estando relacionada com aumento dos índices de morbimortalidade materna e neonatal. Este procedimento está associado com maior risco de infecção puerperal, mortalidade e morbidade materna, prematuridade e mortalidade neonatal. ${ }^{6-7}$

Em relação a mortalidade materna é importante destacar que os números atuais no Brasil são alarmantes quando comparados a outros países, indicando possivelmente falhas nas diretrizes políticas, nos profissionais de saúde e na sociedade como um todo. ${ }^{8}$ Para transformar esta situação é primordial que a assistência baseie-se pelo respeito aos direitos de cidadania das mulheres, desde informações sobre seu estado clínico, as melhores evidências científicas, o acolhimento as suas necessidades e a escuta qualificada. É preciso incluir nos estudos sobre mortalidade materna aspectos que vão além dos dados epidemiológicos. ${ }^{9-10}$

Quanto a mortalidade infantil e neonatal, a literatura sugere que não é a cesariana o fator que influenciou este aspecto, mas sim a melhora na assistência neonatal. Uma das causas mais prevalentes de morte neonatal é a asfixia/hipóxia, sendo estes óbitos evitáveis e refletindo a qualidade da assistência ao parto e ao recémnascido. Sabe-se que, nascidos de cesárea eletiva, quando comparados com os nascidos por parto vaginal, possuem risco maior de morbimortalidade respiratória, sugerindo que o trabalho de parto possui função relevante na maturação pulmonar. ${ }^{11-14}$

Qualificar a assistência ao parto com a implantação das boas práticas obstétricas baseadas em evidências científicas, onde tecnologias sejam utilizadas de forma coerente e de acordo com as necessidades, é uma das alternativas que podem garantir a redução da mortalidade infantil e neonatal, visto que a cesariana por si só não dá conta desta tarefa. ${ }^{14-16}$

Quanto a associação entre prénatal e local de moradia, a literatura identifica a relação entre taxas aumentadas de cesarianas em classes sociais mais altas da sociedade, demonstrando que estes percentuais podem estar refletindo fatores socioculturais na prática obstétrica, bem como fatores institucionais e financeiros. Índices elevados de cesariana estão associados significativamente com aumento da escolaridade materna, maior renda familiar, em regiões com maior percentual de população economicamente ativa e moradoras de locais com melhor qualidade de vida. ${ }^{17}$

As desigualdades socioeconômicas demonstram que a cesariana na verdade vem sendo utilizada nos grupos com melhores condições de saúde materna e menor 


\section{JOURNALOF \\ NURSING \\ AND}

ISSN 2236 - 1987

de políticas públicas regionalizadas e setoriais. Dessa maneira, poder-se-ia trabalhar setorialmente com as necessidades de cada região, flexibilizando protocolos, qualificando profissionais frente às melhores evidências científicas e acolhendo as necessidades das mulheres a partir de suas características sociodemográficas específicas. Além disso, a avaliação em saúde, por meio da setorialização, permitiria um acompanhamento próximo das mudanças e um auxílio às transformações que se mostrarem mais resistentes ou desconexas das políticas públicas de humanização do parto e nascimento atuais.

\section{CONCLUSÕES} de risco, no entanto, autores chamam a atenção para o componente profissional envolvido nesta etapa, sugerindo que o tipo e a qualidade de informações transmitidas durante 0 pré-natal possam influenciar na escolha final do tipo de parto. ${ }^{19-20}$

Assim, a determinação da cirurgia cesariana como via de nascimento é influenciada pela assistência recebida no pré-natal, pela fonte de pagamento, principalmente no caso da saúde suplementar, e por fatores socioeconômicos, sugerindo que esta via de parto é na verdade tratada como um bem de consumo. ${ }^{21}$ Portanto, a modificação deste cenário têm como desafio transformar recomendações e protocolos baseados em evidências científicas em práticas concretas e cotidianas, sem esquecer o tratamento digno, respeitoso e a autonomia das mulheres. ${ }^{19}$

Por fim, ressalta-se a importância destes dados para a compreensão da realidade obstétrica da capital, o que possibilita principalmente a construção
A partir do objetivo proposto neste trabalho de descrever a realidade obstétrica em Porto Alegre, RS, pode-se encontrar um cenário preocupante quando consideram-se as recomendações da OMS principalmente sobre percentuais de cesariana. Ficou claro a partir dos resultados a relação entre nível socioeconômico e pré-natal adequado com 0 tipo de parto vivenciado pelas mulheres porto alegrenses.

Considera-se que, na realidade atual da capital gaúcha, dependendo de onde a mulher mora, pode-se consideravelmente cogitar o tipo de parto que ela terá, independente se atendida pelo setor privado/suplementar ou público. Quanto maior a sua escolaridade, melhores suas condições financeiras, melhor qualidade de vida e, dependendo do seu bairro ou região de moradia, ela possui maiores chances de ter seu filho por via cirúrgica. 


\section{JOURNALOF \\ NURSING \\ AND HEALTH}

Morar nos melhores lugares de Porto Alegre, nas regiões consideradas mais ricas e economicamente ativas é fator predisponente considerável para que a mulher faça uma cesariana na capital. As desigualdades socioeconômicas ficam claras e refletem uma diferenciação nítida de status social na cidade, na medida em que cesarianas não são mais para as mulheres que clinicamente necessitam, mas basicamente para aquelas que possuem condições financeiras para arcar com este procedimento.

Trata-se de um problema de saúde pública que perpassa as questões clínicas e obstétricas e parece estar cercado de uma cultura que tornou a cesariana um desejo de consumo. Parto normal somente nas regiões mais pobres da capital, onde, contraditoriamente, se sobrepõem riscos que poderiam levar à necessidade de uma cesariana.

Em Porto Alegre nascer tem hora marcada para a maioria da população e a escolha do tipo de parto que será realizado possui relação com o lugar em que a mulher está inserida. E este lugar não é apenas uma região, um bairro, mas sim uma produção social permeada por sentimentos, interpretações, memórias que fazem com que os sujeitos estabeleçam suas ações e escolham os caminhos que devem seguir.

Pensando na programação das ações em saúde, conclui-se que nas regiões mais nobres do município são necessárias ações de informação e educação em saúde junto às mulheres, focadas nas melhores evidências científicas sobre o parto normal e sobre as indicações clínicas para a
ISSN 2236 - 1987

cirurgia cesariana, bem como sobre os riscos e benefícios de ambos os tipos de cuidado. Além disso, indica-se, com certa urgência, a realização de ações educacionais e de qualificação por parte dos gestores junto aos profissionais do subsetor privado, no intuito de disseminar o conhecimento e os protocolos assistências que garantam a segurança e a qualidade técnico-científica de suas ações.

Já no setor público é preciso, além de ações de educação em saúde das mulheres com foco nas evidências científicas, um enfrentamento das condições de saúde que possam refletir nos índices de desenvolvimento humano e na mortalidade materna e infantil, investindo-se na cobertura pré-natal e na qualidade do cuidado prestado.

Por fim, reflete-se sobre as limitações desse estudo, as quais estão relacionadas com sua metodologia, já que se considerou apenas dados brutos de sistemas de informação públicos, muitas vezes desatualizados e que, por si só, não refletem a realidade vivida pelos serviços de saúde e pelas mulheres porto-alegrenses. É preciso utilizar-se de mais dados e realizar análises estatísticas mais aprofundadas, que permitam associações de diversos tipos, para que conclusões mais profundas sobre a realidade obstétrica sejam demonstradas. Além disso, torna-se importante ouvir profissionais atuantes neste setor e mulheres que vivenciam esta realidade para que as políticas públicas possam ser construídas não somente sobre dados brutos e números, mas principalmente sobre a realidade vivida no cotidiano dos sujeitos. 


\section{NURSING \\ AND \\ HEA}

Assim, a partir dos dados aqui levantados e das limitações apresentadas, abrem-se possibilidades para novas pesquisas com objetivos e temáticas que possibilitem a visibilidade da realidade vivida na área materno e neonatal e o incremento de políticas públicas que se aproximem da humanização da assistência e da integralidade do cuidado com as mulheres, bebês e famílias.

\section{REFERÊNCIAS}

1. Nakano AR, Bonan C, Teixeira LA. A normalização da cesárea como modo de nascer: cultura material do parto em maternidades privadas no Sudeste do Brasil. Physis (Rio J). 2015;25(3):885-904.

2. Leal MC, Gama SGN. Nascer no Brasil. Cad saúde pública. 2014;30 Suppl:S5-7.

3. Velho MB, Santos EKA, Collaço VS. Parto normal e cesárea: representações sociais de mulheres que os vivenciaram. Rev bras enferm. 2014;67(2):282-9.

4. Paris GF, Monteschio LVC, Oliveira RR, Latorre MRDO, Pelloso SM, Mathias TAF. Tendência temporal da via de parto de acordo com a fonte de financiamento. Rev bras ginecol obstet. 2014;36(12):548-54.

5. Prefeitura Municipal de Porto Alegre. OBSERVAPOA. Porto Alegre em Análise: sistema de gestão e análise de indicadores. Porto Alegre, 2017. [Internet]. [acesso 2016 mar 07]. Disponível em: http://portoalegreemanalise.procemp a.com.br/

6. Gonçalves IS, Lopes S, Casanova J, Fortuna J, Silva PT. Impact of
ISSN $2236-1987$

strategies in reducing cesarean section rate. Acta obstétrica e ginecológica portuguesa. 2014;8(1):29-35.

7. Victora CG, Aquino EML, Leal MC, Monteiro CA, Barros FC, Szwarcwald CL. Maternal and child health in Brazil: progress and challenges. The lancet. 2011;377(9780):1863-76.

8. Dias $M A B$, Domingues RMSM, Schilithz ACO, Nakamura-Pereira $M$, Diniz CSG, Brun IR, et al. Incidência do near miss materno no parto e pós-parto hospitalar: dados da pesquisa Nascer no Brasil. Cad saúde pública. 2014;39 Suppl:S169-81.

9. Rezende CB. The Experience of Pregnancy Subjectivity and social relations. Vibrant (Brasília). 2011; 8(2):529-49.

10. Rezende CB. O parto em contexto Narrativas da gravidez entre gestantes no Rio de Janeiro. Civitas: revista de ciências sociais. 2015;15(2):214-28.

11. Carneiro JA, Costa FM, Vieira MM, Reis TC, Carneiro MAC, Caldeira AP. Characteristics of newborn of very low weight admitted to intensive neonatal care unit. J nurs UFPE on line [Internet]. 2015;9(4):7207-12. Disponível em: https: / / periodicos.ufpe. br/revistas/r evistaenfermagem/article/download/ $10480 / 11327$

12. Hansen AK, Wisborg K, Uldbjerg N, Henriksen TB. Risk of respiratory morbidity in term infants delivered by elective caesarean section: cohort study. BMJ. 2008;336:85-7.

13. Daripa M, Caldas HMG, Flores LPO, Waldvogel BC, Guinsburg R, Almeida MFB. Asfixia perinatal associada à mortalidade neonatal precoce: estudo 


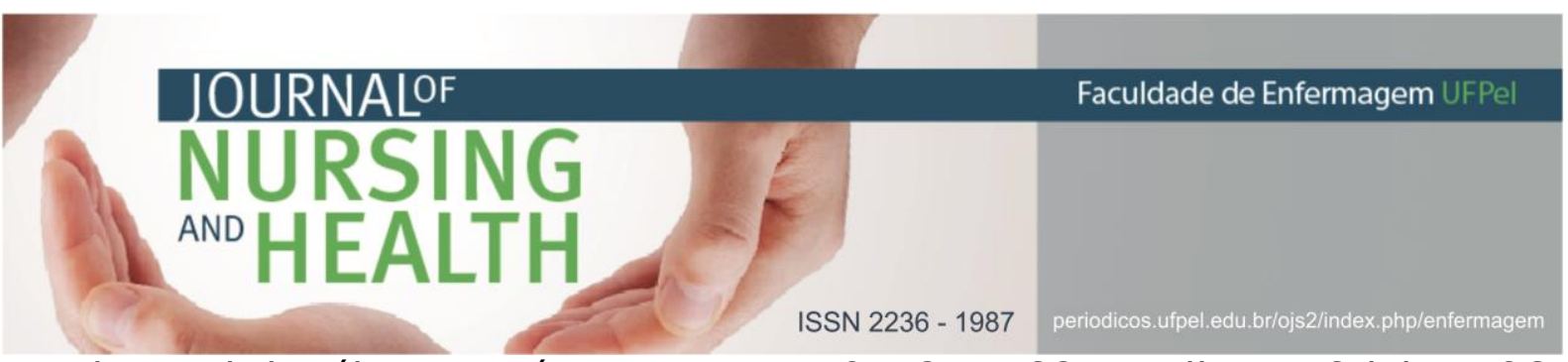

populacional dos óbitos evitáveis. Rev 21. Gama SGN, Viellas EF, Schilitz AOC, paul pediatr. 2013;31(1):37-45.

14. Lansky S, Friche MAL, Silva AAM, Campos D, Bittencourt SDA, Carvalho $M L$, et al. Pesquisa Nascer no Brasil: perfil da mortalidade neonatal e avaliação da assistência à gestante e ao recém nascido. Cad saúde pública. 2014;30 Suppl:S1-15.

15. Cortês CT, Santos RCS, Caroci AC, Oliveira SG, Oliveira SMJ, Riesco MLG. Metodologia de implementação de práticas baseadas em evidências científicas na assistência ao parto normal: estudo piloto. Rev esc enferm USP.2015;49(5):716-25.

16. Ministério da Saúde (BR). Agência Nacional de Saúde Suplementar. O modelo de atenção obstétrica no setor de Saúde Suplementar no Brasil: cenários e perspectivas. Brasília; 2008.

17. Freitas PF, Savi EP. Desigualdades sociais nas complicações da cesariana: uma análise hierarquizada. Cad saúde pública. 2011;27(10):2009-20.

18. Souza Junior JCS, Kunkel N, Gomes MA, Freitas PF. Equidade inversa e desigualdades no acesso à tecnologia no parto em Santa Catarina, Brasil, 2000 a 2004. Rev bras saúde mater infant. 2007;7(4):397-403.

19. Domingues RMSM, Dias MAB, Nakamura-Pereira M, Torres JÁ, Dorsi $E$, Pereira APE, et al. Processo de decisão pelo tipo de parto no Brasil: da preferência inicial das mulheres à via de parto final. Cad saúde publica.2014;30 Suppl:S101-116.

20. Pádua KS, Osis MJD, Faúndes $A$, Barbosa AH, Moraes Filho OB. Fatores associados à realização de cesariana em hospitais brasileiros. Rev saúde pública. 2010;44(1):70-9. 